\title{
Artiflex Toric Phakic Intraocular Lens Implantation in Congenital Nystagmus
}

\author{
Gonzalo Muñoz ${ }^{\mathrm{a}}$ Lurdes Belda ${ }^{\mathrm{a} b}$ César Albarrán-Diego \\ Teresa Ferrer-Blasco ${ }^{\text {b }}$ Santiago García-Lázarob \\ aRefractive Surgery Department, Centro Oftalmológico Marqués de Sotelo and \\ Hospital NISA Valencia al Mar, and ${ }^{b}$ Optometry Research Group, University of \\ Valencia, Valencia, Spain
}

\section{Key Words}

Nystagmus · Refractive surgery · Toric phakic intraocular lens

\begin{abstract}
Case: A 44-year-old woman with congenital nystagmus and myopic astigmatism in both eyes who was submitted to phakic intraocular lens ( $\mathrm{plOL}$ ) implantation.

Methods: Full ophthalmologic examination including refractive status, corrected (CDVA) and uncorrected (UCVA) monocular and binocular visual acuities, ocular motility, slitlamp evaluation, tonometry and fundoscopy before and after implantation of toric plOLs (Artiflex; Ophtec BV, The Netherlands) in both eyes.

Results: Preoperative logMAR CDVA were 0.699 and 0.420 in the right and left eye, respectively. Three months after surgery, logMAR UCVA were 0.398 and 0.182 , reaching binocular logMAR UCVA of 0.132 . There were no changes in nystagmus characteristics after surgery.
\end{abstract}

Conclusions: plOL implantation may be a safe and suitable treatment to correct high refractive errors in patients with congenital nystagmus. Significant improvement in CDVA and UCVA can be achieved.

\section{Introduction}

Nystagmus is an ocular motor disorder characterized by involuntary and rhythmic movements of one or both eyes [1]. Nystagmus can be classified according to the age of onset (congenital or acquired), direction (horizontal, vertical, torsional or combinations) or considering the character of movements (jerk, pendular, dual jerk) [1,2]. Patients with nystagmus usually have a diminished visual performance because of these eye movements. This includes deficiencies in visual acuity inversely proportional to the 
intensity of nystagmus [3], and anomalies in color vision, contrast sensitivity and stereopsis [2]. The more common clinical signs and symptoms are amblyopia, anomalous head posture, oscillopsia, photophobia and hypoaccommodation $[1,2]$.

Different ways to correct refractive errors in patients with nystagmus can be found, grouped into non-surgical and surgical procedures. Those related to non-surgical treatments include spectacles [1,2] and contact lenses [3]. On the other hand, laser corneal refractive surgery has been recently applied as an alternative to treat these patients [4]. However, to the best of our knowledge, no previous reports regarding the use of phakic intraocular lenses (pIOLs) for correcting refractive errors in patients with nystagmus has been reported. The present study reports the case of a patient with congenital nystagmus submitted to Artiflex toric pIOL (Ophtec BV, The Netherlands) implantation for refractive error correction.

\section{Case Report}

A 44-year-old woman with congenital nystagmus visited our center aiming to obtain spectacle independence. She had been wearing contact lenses for many years, but she has suffered from dryness for 2 years and discontinued lens wear. Her manifest refraction was $-8.50-3.50 \times 20^{\circ}$ in her right eye $(\mathrm{RE})$ and $-8.00-3.50 \times 160^{\circ}$ in her left eye (LE). Corrected distance visual acuity (CDVA) using spectacles was $0.699 \operatorname{logMAR}(\mathrm{RE})$ and $0.420 \log$ MAR (LE). The patient displayed a horizontal jerk nystagmus $10^{\circ}$ in amplitude. The nystagmus frequency was moderate and increased with attempted fixation. No well-defined null zone or anomalous head movement or position could be determined. Preoperative examination included manifest refraction, CDVA using spectacles, slit-lamp biomicroscopy, Goldman tonometry, binocular indirect ophthalmoscopy, biometry, pachymetry, keratometry and corneal topography (table 1). pIOL implantation surgery was explained, and written informed consent was obtained.

The Artiflex toric pIOL consists of a three-piece lens made up of hydrophobic polysiloxane optic and polymethylmethacrylate rigid haptics. It is an iris-fixated foldable pIOL with a 6-mm optical zone diameter, designed for the correction of regular myopic astigmatism. Refractive power sphere varies from -1.0 to -13.5 diopters $(\mathrm{D})$ in combination with a cylinder correction from -1.0 to $-5.0 \mathrm{D}$. Furthermore, the Artiflex toric pIOL claw design guarantees long-term rotational stability in addition to avoiding endothelium-lens contact. The power of the pIOL was calculated using the van der Heijde formula, which uses vertex distance, subjective spectacle refraction, corneal curvature and anterior chamber depth.

Both eyes were treated by the same surgeon (G.M.) within a 24-hour period. Surgery was carried out under peribulbar anesthesia. The Artiflex toric pIOL was implanted through a 3.2-mm clear-cornea incision located at 12 o'clock in both eyes. The marking technique previously reported by Graether [5] was used, consisting of placing a single ink mark at the limbus at $6 o^{\prime}$ clock with the patient in the upright sitting position during the preoperative preparation. A $360^{\circ}$ gauge was applied onto the eye having the patient lying back, and aligning the $90-270^{\circ}$ axis on the mark at the $60^{\prime}$ clock position previously made to compensate for a postural axis shift. A half-circle axis marker was placed so that the index mark on the semicircle was aligned with the minus axis on the cornea $\left(20-200^{\circ}\right.$ in the RE and $160-340^{\circ}$ in the LE), which was the axis where the Artiflex toric pIOL had to be fixated. Two 1-mm side port incisions were done at the appropriate axis to fixate the PIOL to the iris by means of forceps. At the end of the procedure, a single 10/0 nylon suture was used to secure the incision in both eyes. There were no complications in the surgical procedures. Postoperative treatment included tobramycin and dexamethasone eyedrops (TobraDex, AlconLabs) 4 times daily for 7 days and then tapering it slowly during the following 3 weeks. Lubricant eyedrops (Acuolens, AlconLabs) were prescribed 3-4 times daily for 3 months.

Postoperative examinations included monocular and binocular uncorrected distance visual acuity (UCVA), manifest refraction, CDVA and intraocular pressure (IOP) measurements performed at 1 day, 1 week and 1 and 3 months after surgery. No postoperative complications appeared including high IOP. 
The slit-lamp examination showed no pIOL rotation, they were well-centered in both eyes (fig. 1). UCVA at 3 months of follow-up were 0.398 (logMAR) and 0.182 (logMAR) for the RE and LE respectively, with binocular UCVA of 0.132 (logMAR), while best spectacle-corrected visual acuity was $0.378(\log M A R)$ with $-0.75 \times 60^{\circ}$ in her RE and $0.155(\log M A R)$ with $-0.75 \times 150^{\circ}$ in her LE. Postoperative binocular UCVA at 3 months was 0.132 (logMAR).

\section{Ethics}

All study procedures adhered to the recommendations of the declaration of Helsinki.

\section{Discussion}

Nystagmus is an ocular motor disorder with repetitive and involuntary oscillations of the eye. It is usually associated to visual dysfunctions and vision impairment including significant amblyopia. Different optical and surgical treatment modalities have been proposed in order to reduce oscillations and improve visual performance $[1,2]$.

Correction of significant refractive error in patients with nystagmus is the single most powerful intervention to improve visual functions [2]. The use of contact lenses has also been described in patients with nystagmus [3]. Contact lenses correct refractive errors moving simultaneously along with the eye and providing a more continuous fixation than spectacles, reducing higher-order optical aberrations together with the prismatic effect. Allen and Davies [3] evaluated the use of contact lenses in patients with nystagmus, reporting significant improvement of 2 lines of visual acuity and better contrast sensitivity in comparison with spectacles.

Recently, laser corneal refractive surgery has been used in patients with nystagmus [4]. As previously stated, visual acuity in nystagmus is often poor due to the extremely short foveation time, making these patients theoretically poor candidates for laser corneal refractive surgery. Previous authors consider nystagmus a contraindication to laser corneal refractive surgery because of the involuntary ocular movements which might cause decentered ablation and problems associated to the suction system for creating the flap. However, new laser systems use high-speed active eye-tracking which may counteract eye motion during laser ablation. In addition, suction systems to fixate the globe during flap preparation seem to be effective to avoid flap-creation problems in these patients. For instance, Mahler et al [4] found good outcomes in 8 patients with nystagmus (CDVA improved in $62.5 \%$ of the eyes). They concluded that LASIK surgery may be safely performed in nystagmus using an active tracking system at $120 \mathrm{~Hz}$ with or without mechanical fixation.

Patients with nystagmus may display high refractive errors that could contraindicate laser corneal surgery, making pIOLs the alternative of treatment. Similarly, a recent report from the American Academy of Ophthalmology concluded that in cases of myopia levels equal to or higher than $-8 \mathrm{D}$, pIOLs may provide a better visual outcome than keratorefractive surgery [6]. Up until now, no reports have been available in the literature considering pIOLs in patients with nystagmus.

A case of a young patient with nystagmus aiming to obtain spectacle independency is presented here. This patient was submitted to Artiflex toric pIOL implantation in both eyes. After the surgery, the patient had no visual complaints and achieved an UCVA 
better than preoperative spectacles CDVA, improving 2 and 3 lines of visual acuity in the $\mathrm{RE}$ and LE, respectively. When comparing preoperative and postoperative visual acuity, it should be considered that normally a change in magnification of the retinal image occurs. For myopes who are corrected with spectacles, the magnification is less than one. For example in the present case, a -8.00-D myopic eye corrected in the 16- $\mathrm{mm}$ spectacle plane would get around $15 \%$ magnification after pIOL surgery. Thus, if no other effects were involved, visual acuity should improve about 2-3 lines after surgery for myopia, and the effect will obviously increase with the magnitude of the correction. This increase in visual acuity agrees with the change in visual acuity between before and after surgery reported for both eyes in the present patient. Similar effects would be found considering laser corneal refractive surgery, but corneal refractive surgery increases optical aberrations of the eye as well, therefore the acuity increase would be smaller than that predicted by magnification alone, and better outcomes might be expected when a PIOL is implanted. Small residual refractive errors occurring after pIOL implantation may be corrected using laser corneal surgery, which has been proved to be safe and effective combined with pIOLs [7].

No sight-threatening and visual complications were observed in the present study: high IOP, halos, glare, inflammation (e.g. acute uveitis), cataracts or pigment deposits, for example. One of the main problems with anterior chamber pIOL implantation is the potential for damage to the anterior segment structures, especially the corneal endothelium. However, previous studies indicate that new models of angle-supported and iris-fixated pIOLs are well tolerated by the corneal endothelium as improvement in IOL design and quality of surgery is achieved [8]. In addition, as the Artiflex pIOL has no angular support, the possibility of peripheral contact with the corneal endothelium is diminished. Unlike posterior chamber pIOLs, the Artiflex IOL is located far from the crystalline lens, thus decreasing the possibility of cataract complication.

One interesting factor to consider in contact lens and surgical procedures (laser corneal refractive surgery or pIOL implantation) is the fact that the patient is looking along the visual axis of his correction for a far greater proportion of the time than with spectacles, since the correction moves with the eye. This becomes particularly important for the patient with high refractive error. It may be suggested that pIOL implantation can diminish the amplitude and frequency of the nystagmus, considering that the mechanism for this reduction might be the additional vergence and accommodative effort generated by the pIOL. In the present case, the amplitude and frequency of the nystagmus was not changed after pIOL surgery. In addition, it is not expected that ocular movement due to the nystagmus will affect the stability of the pIOL in a short-medium period of time. A large sample and longer follow-up would be necessary, however.

In conclusion, this case report shows that PIOL implantation can be a safe therapeutic option to correct high refractive errors in patients with nystagmus. Some of these patients may greatly benefit from the surgery, which may improve their visual acuity and quality of life.

\section{Disclosure Statement}

The authors indicate no financial support or financial conflict of interest. 
Table 1, Patient characteristics before and after surgery

\begin{tabular}{lll}
\hline & Right eye & Left eye \\
\hline Preoperative & & \\
ACD, mm & 3.55 & 3.55 \\
AL, mm & 27.24 & 26.67 \\
Keratometry, D & & \\
$\quad$ K1 & 42.62 & 42.75 \\
$\quad$ K2 & 46.25 & 46.00 \\
BCVA, logMAR & 0.699 & 0.420 \\
Refraction, D & $-8.50-3.50 \times 20^{\circ}$ & $-8.00-3.50 \times 160^{\circ}$ \\
pIOL power, D & $-9.00-3.50 \times 0^{\circ}$ & $-8.50-3.50 \times 0^{\circ}$ \\
Axis pIOL implantation, ${ }^{\circ}$ & 20 & 160 \\
\hline Postoperative & & \\
UCVA, logMAR & 0.398 & 0.182 \\
BCVA, logMAR & 0.357 & 0.155 \\
Refraction, D & $-0.50 \times 60^{\circ}$ & $-0.50 \times 150^{\circ}$ \\
\hline
\end{tabular}

$\mathrm{ACD}=$ Anterior chamber depth; $\mathrm{AL}=$ axial length $\mathrm{BCVA}=$ best spectacle-corrected visual acuity; $\mathrm{PIOL}=$ phakic intraocular lens; $\mathrm{UCVA}=$ uncorrected distance visual acuity.

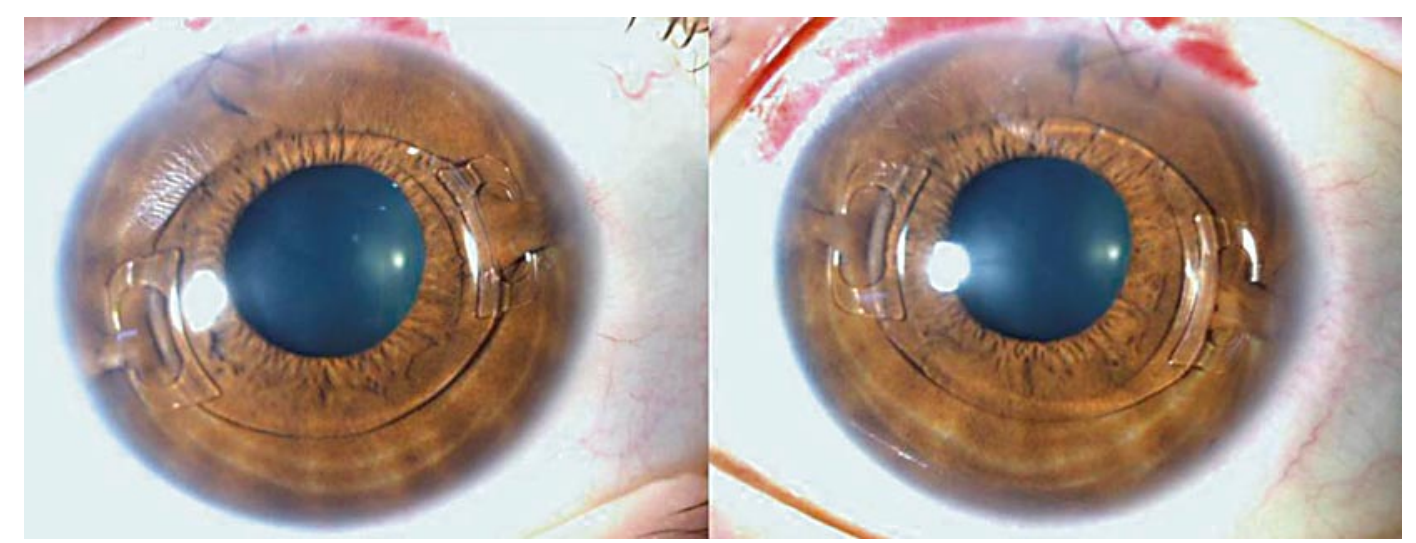

Fig. 1. Artiflex toric phakic intraocular lens centered in the right and left eye. 


\section{References}

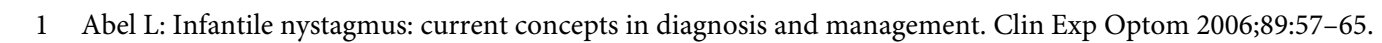

$\checkmark 2$ Hertle RW: Examination and refractive management of patients with nystagmus. Surv Ophthalmology 2000;45:215-222.

3 Allen ED, Davies PD: Role of contact lenses in the management of congenital nystagmus. Br J Ophthalmol 1983;67:834-836.

4 Mahler O, Hirsh A, Kremer I, et al: Laser in situ keratomileusis in myopic patients with congenital nystagmus. J Cataract Refract Surg 2006;32:464-467.

5 Graether JM: Simplified system of marking the cornea for a toric intraocular lens. J Cataract Refract Surg 2009;35:1498-1500

-6 Huang D, Schallhorn SC, Sugar A, Farjo AA, Majmudar PA, Trattler WB, Tanzer DJ: Phakic intraocular lens implantation for the correction of myopia: a report by the American Academy of Ophthalmology. Ophthalmology 2009;116:2244-2258.

7 Muñoz G, Alió JL, Montés-Micó R, Belda JI: Angle-supported phakic intraocular lenses followed by laserassisted in situ keratomileusis for the correction of high myopia. Am J Ophthalmol 2003;136:490-499.

-8 Alió JL, de la Hoz F, Perez-Santonja JJ, et al: Phakic anterior chamber lenses for the correction of myopia: a 7year cumulative analysis of complications in 263 cases. Ophthalmology 1999;106:458-466. 